\section{Tamanho ao nascer e problemas de saúde mental aos 11 anos em uma coorte brasileira de nascimentos}

\author{
Size at birth and mental health problems \\ at 11 years of age in a Brazilian birth cohort
}

\author{
Erika Alejandra Giraldo Gallo ${ }^{1}$ \\ Luciana Anselmi 1 \\ Samuel C. Dumith 1 \\ Marcia Scazufca 1 \\ Ana M. B. Menezes 1 \\ Pedro C. Hallal 1,2 \\ Alicia Matijasevich 1
}

\title{
Introdução
}

1 Faculdade de Medicina, Universidade Federal de Pelotas, Pelotas, Brasil. 2 Grupo de Estudos em Epidemiologia da Atividade Física, Universidade Federal de Pelotas, Brasil.

\section{Correspondência} A. Matijasevich Departamento de Medicina Social, Faxuldade de Medicina, Universidade Federal de Pelotas.

Rua Marechal Deodoro 1160 30 andar, Pelotas, RS 96020-220, Brasil. amatija@yahoo.com

\begin{abstract}
The aim of this study was to evaluate the association between size at birth and mental health problems at 11 years of age in the 1993 Pelotas (Brazil) Birth Cohort Study. Newborns were weighed and measured, and anthropometric indices were calculated. At 11 years of age, mental health problems were assessed using the Strengths and Difficulties Questionnaire (SDQ). Prevalence of mental health problems was $32 \%$ (95\%CI: 31-33). After adjusting for potential confounders, newborns with weight and body mass index (BMI) for age $z$-scores $<-2$ SD were at $27 \%$ (95\%CI: 7-49) and 29\% (95\%CI: 10-51) greater risk, respectively, of developing mental health problems at age 11 years than those born with normal scores. Newborns with BMI and head circumference for age $z$-scores $>+2 S D$ were at $34 \%$ (95\%CI: 6-71) and 19\% (95\%CI: 1-40) greater risk, respectively, of developing mental health problems than those with normal scores. The results suggest that early factors that are reflected as size measurements at birth can cause mental health problems later in life.
\end{abstract}

Fetal Development; Mental Health; Anthropometry
Os estudos sobre as origens do desenvolvimento das doenças têm evidenciado que o feto responde às mudanças ambientais. Os órgãos e sistemas do corpo humano passam por diferentes períodos críticos, ocorridos especialmente durante o desenvolvimento fetal ${ }^{1}$. Alterações durante esses períodos podem gerar consequências desfavoráveis permanentes na estrutura e na fisiologia do indivíduo ao longo da vida 2 . A plasticidade do desenvolvimento permite ao feto adaptar-se às condições ambientais no útero e responder a estas com restrições que permitam a sobrevivência após o nascimento ${ }^{3}$.

Diversos estudos na epidemiologia do ciclo vital têm evidenciado associação do baixo peso ao nascer (BPN) com doenças crônicas na vida adulta, como doença coronariana, acidente vascular cerebral, hipertensão e diabetes tipo 2 2,3,4. De acordo com a hipótese da programação fetal, proposta por Barker 1, chamada atualmente de "Origens do Desenvolvimento da Saúde e das Doenças" (Developmental Origins of Health and Disease), exposições ambientais durante esses períodos críticos podem afetar não só a susceptibilidade e/ou o risco de desenvolver distúrbios fisiológicos e metabólicos, como também condicionar os padrões de comportamento e a resposta aos efeitos adversos durante a vida adulta.

Fatores que afetam o crescimento na vida intrauterina ou na infância precoce podem in- 
fluenciar o desenvolvimento neurológico. Estudos em animais demonstraram que a desnutrição em etapas precoces da vida afeta a estrutura e função cerebral, levando a uma alteração das funções cognitivas e dos processos funcionais psicológicos relacionados 5,6, o que posteriormente foi demonstrado em seres humanos 7 . A redução da atividade da função no eixo hipotálamo-hipófise-glândulas suprarenais (HPAA) é característica importante de várias doenças relacionadas ao estresse e depressão, o que sugere que a susceptibilidade para o desenvolvimento destas doenças seja originada pelas alterações sofridas durante as primeiras etapas da vida 8 .

Vários estudos documentaram a associação entre restrição do crescimento fetal e o risco de desenvolver transtornos do comportamento, esquizofrenia e psicoses na adolescência ou na vida adulta 9,10,11,12. O peso ao nascer, nesse sentido, é um indicador da saúde do recém-nascido, que reflete as condições da vida intrauterina. Logo, o BPN é um indicador proxy das restrições sofridas durante o desenvolvimento fetal e um dos melhores preditores da mortalidade neonatal e infantil. Saigal 13 observou que crianças com BPN têm 40 vezes mais riscos de morrer no período neonatal e de apresentar taxas mais elevadas de morbidade e sequelas no seu desenvolvimento físico e intelectual. Outros estudos encontraram associação entre BPN e problemas psicológicos, tais como transtornos de ansiedade, hiperatividade e déficits de atenção, problemas de comportamento, sintomas semelhantes a psicoses, transtornos afetivos e suicídio 14,15,16,17,18.

Estudos realizados nas coortes de Helsinki (Finlândia) evidenciaram que os problemas de externalização ou do comportamento disruptivo, tais como déficit de atenção e hiperatividade, estiveram associados ao baixo índice ponderal, pequeno perímetro cefálico e déficit de perímetro cefálico para comprimento ao nascer 19. Da mesma forma, condutas de hostilidade na vida adulta foram relacionadas ao BPN e ao déficit do índice de massa corporal (IMC) ao nascimento 20. O estudo da coorte de nascimentos Avon Longitudinal Study of Parents and Children (ALSPAC), mostrou associação inversa entre comprimento ao nascimento e problemas de saúde mental aos sete anos 9 .

Há poucas pesquisas no contexto do ciclo vital que tenham estudado a associação entre distúrbios do crescimento fetal e o tamanho ao nascimento com o desenvolvimento de problemas de saúde mental em adolescentes e préadolescentes de países de renda média ou baixa. Muitas pesquisas têm estudado só o BPN, e poucas estudaram comprimento, perímetro cefálico ou outras medidas ou índices antropométricos ao nascimento e sua associação com problemas de saúde mental em etapas mais tardias.

O objetivo do presente estudo foi analisar a associação entre o tamanho ao nascimento e problemas de saúde mental aos 11 anos nos indivíduos da Coorte de Nascimentos de Pelotas do ano de 1993. Este trabalho vem contribuir para a discussão de que eventos ocorridos durante a gestação e nascimento podem afetar a saúde mental em etapas posteriores, colaborando para o planejamento de estratégias de saúde pública que permitam a prevenção precoce das doenças mentais.

\section{Métodos}

Pelotas é uma cidade situada no Estado do Rio Grande do Sul, localizada ao extremo sul do Brasil, com uma população estimada de 345.179 habitantes, onde $93 \%$ de seus moradores residem na zona urbana 21 . No ano de 1993, foi iniciado o estudo de coorte de nascimentos de Pelotas que incluiu todos os nascimentos ocorridos nas cinco maternidades da cidade, entre 1o de janeiro e 31 de dezembro do referido ano (mais de $99 \%$ dos partos foram hospitalares). Os 5.249 recém-nascidos, cujas mães eram residentes da zona urbana e que aceitaram participar do estudo, foram incluídos na coorte. A metodologia detalhada desse estudo pode ser consultada em publicações anteriores 22,23.

Durante a pesquisa perinatal, as mães foram entrevistadas para coletar informações demográficas e socioeconômicas da família, características da gestação, hábitos maternos (tabagismo e consumo de álcool), utilização de serviços de saúde e informações referentes ao pré-natal e parto. Os recém-nascidos foram pesados e medidos nas primeiras 24 horas. O peso ao nascer foi medido com balanças pediátricas de mesa (CMS Weighing Equipment Ltd., Londres, Inglaterra), calibradas periodicamente (precisão de 10g). Para o comprimento ao nascer, usou-se infantômetros ARTHAG (Appropriate Health Resources and Technologies Action Group, Londres, Inglaterra) na posição supina (precisão de $1 \mathrm{~mm}$ ) e para obter o perímetro cefálico utilizou-se fita métrica inelástica (precisão de $1 \mathrm{~mm}$ ). Todas as medidas foram obtidas mediante técnicas padronizadas em treinamento.

No ano de 2004, quando os indivíduos pertencentes à coorte tinham uma idade média de 11,3 anos (mínimo: 10,6 anos e máximo: 12,2), foi realizado um novo acompanhamento que permitiu localizar e coletar as informações de 87,5\% destes, a fim de estudar os aspectos da saúde e da vida dos membros da coorte e de suas famílias 22 . 
Os problemas de saúde mental dos indivíduos do estudo foram avaliados durante o seguimento feito no ano de 2004. Usou-se o Questionário de Capacidades e Dificuldades (Strengths and Difficulties Questionnaire - SDQ) 24,25. O SDQ é um instrumento de rastreamento que permite detectar possíveis problemas de saúde mental em pessoas com idades entre 4 e 16 anos. De acordo com o autor desse instrumento, foram incluídos na categoria de "problemas de saúde mental" os sintomas emocionais, de desatenção e hiperatividade, de conduta e os problemas de socialização com os pares 26. O SDQ possui três versões: (a) para pais; (b) para professores; e (c) para os próprios adolescentes. O questionário consta de 25 perguntas referidas ao comportamento da criança ou adolescente nos últimos seis meses. A pontuação total de dificuldades foi gerada pela soma dos resultados das subescalas de condutas problemáticas (sintomas emocionais, de desatenção e hiperatividade, de conduta e os problemas de socialização com os pares), conforme instruções localizadas na página da Internet do SDQ 26. No presente estudo foram usados os resultados da versão dos pais, aplicada à mãe do adolescente (ou responsável) em forma de entrevista dirigida por pessoal treinado. Foram considerados indivíduos com problemas de saúde mental aqueles que obtiveram 17 ou mais pontos no escore total do SDQ (somatório das subescalas de dificuldades do SDQ), conforme validação realizada no Brasil por Fleitlich-Bilyk \& Goodman 24.

Analisaram-se como possíveis fatores de risco para problemas de saúde mental as seguintes variáveis coletadas durante o estudo perinatal: índices antropométricos ao nascimento (escores-z de peso, comprimento, perímetro cefálico e IMC para idade) calculados de acordo com as curvas de crescimento publicadas pela Organização Mundial da Saúde (OMS) em 2006 27; restrição do crescimento intrauterino (RCIU, crianças com peso ao nascer inferior ao percentil 10 para sua idade gestacional e sexo segundo a população de referência de Kramer et al. 28); índice ponderal (peso/comprimento ${ }^{3}$ ) e peso ao nascer em três categorias (<2.500g; 2.500-3.499g; $\geq 3.500 \mathrm{~g}$ ). Crianças com escores-z abaixo de -2 desviospadrão (DP) em qualquer dos indicadores foram consideradas com déficit, e aquelas com escores- $\mathrm{z}$ acima de +2 DP foram consideradas com excesso.

Os potenciais fatores de confusão da associação entre tamanho ao nascer com os problemas de saúde mental aos 11 anos foram coletados durante o estudo perinatal: renda familiar $(<1$; 1,1-3,0; 3,1-6,0; > 6,1-10,0; > 10,0 salários mínimos); escolaridade materna $(0 ; 1-4 ; 5-8 ; \geq 9$ anos completos de educação formal); idade materna
( $\leq 19 ; 20-34 ; \geq 35$ anos completos no momento do parto); cor da pele da mãe observada pela entrevistadora (branca; negra ou mestiça); situação conjugal da mãe (sem marido ou companheiro; com marido ou companheiro); fumo na gravidez (sim, fumou um ou mais cigarros durante a gravidez; não, nunca fumou na gravidez); consumo de álcool na gravidez (sim, tomou alguma bebida alcoólica durante a gravidez; não, nunca tomou); paridade (número de partos anteriores ao atual, sendo: 0 = primípara; 1 = já teve um parto anterior; $\geq 2$ = multípara); sexo da criança (masculino; feminino); tipo de parto (vaginal; cesariana) e idade gestacional (avaliada usandose o método de Dubowitz et al. 29 e categorizada em: $<34$; 34-36; $\geq 37$ semanas completas de gestação). Categorizou-se como nascimento prétermo aquela criança que apresentou uma idade gestacional menor que 37 semanas ao nascer. A saúde mental da mãe foi avaliada no ano de 2004, durante o acompanhamento dos 11 anos, utilizando-se o instrumento Self Report Questionnaire (SRQ-20) aplicado a todas as mães dos indivíduos da coorte 30,31. Considerou-se um SRQ positivo quando o SRQ da mãe foi $\geq 8$ pontos conforme validação realizada no Brasil por Mari \& Williams 30.

As análises descritivas incluíram o cálculo de distribuições de frequência para variáveis categóricas, medidas de tendência central e dispersão para variáveis numéricas. Modelos de regressão de Poisson com variância robusta foram usados para investigar a associação existente entre o desfecho dicotômico (problema de saúde mental: sim/não) e as variáveis explanatórias. A análise multivariável foi realizada usando-se dois modelos: (1) análise ajustada para os potenciais fatores de confusão coletados no período perinatal: renda familiar, escolaridade da mãe, idade materna ao nascimento, cor da pele da mãe, estado civil, fumo e consumo de álcool durante a gravidez, paridade, sexo da criança, tipo de parto e idade gestacional; e (2) ajustando para o modelo 1 com a adição da variável "saúde mental da mãe" coletada no acompanhamento dos 11 anos.

Foram excluídas do presente trabalho as crianças de partos gemelares. A análise foi conduzida usando-se o programa Stata versão 11.0 (Stata Corp., College Station, Estados Unidos).

O estudo da coorte de nascimentos de 1993 teve a aprovação do Comitê de Ética em Pesquisa da Universidade Federal de Pelotas. Foi obtido Termo de Consentimento Livre e Esclarecido de todas as mães participantes em cada acompanhamento. 


\section{Resultados}

Do total de 5.249 crianças que foram incluídas no estudo inicial, 141 morreram entre o nascimento e os 11 anos. No acompanhamento de 2004, foram entrevistados 4.452 indivíduos, resultando em $12,5 \%$ de perdas. No total, 4.358 pessoas apresentaram informações válidas, tanto do estudo perinatal quanto do questionário SDQ aos 11 anos. As perdas não foram diferentes às da população inicial em relação às variáveis sexo e peso ao nascimento, mas foram mais frequentes entre filhos de mães com renda familiar superior a 6 salários mínimos e com 9 ou mais anos de escolaridade 23

A variável sexo não se comportou como um fator modificador de efeito da associação entre as características antropométricas estudadas e os problemas de saúde mental aos 11 anos (valor de p das interações entre 0,5 e 0,9). Por essa razão, as tabelas apresentam informação conjunta de meninos e meninas.

As Tabelas 1 e 2 mostram a descrição geral dos indivíduos estudados; a primeira conforme as características maternas, e a segunda de acordo com as características das crianças ao nascimento. Observou-se um predomínio de crianças pertencentes a famílias de renda menor que 3 salários mínimos $(61,7 \%)$ e de crianças com mães que tinham as seguintes características: escolaridade entre 5-8 anos (48\%), cor branca (77\%), idade entre $20-34$ anos $(71,4 \%)$ e vivendo com marido ou companheiro $(88,1 \%)$. Verificou-se ainda que $33,2 \%$ das mães das crianças fumaram, $5 \%$ consumiram álcool durante a gravidez e $36,9 \%$ foram multíparas. Aproximadamente $30 \%$ dos indivíduos nasceram por cesariana, 8,4\% apresentaram peso ao nascer $<2.500 \mathrm{~g}, 10,3 \% \mathrm{ti}-$ veram idade gestacional < 37 semanas e $23,2 \%$ apresentaram RCIU. Ao nascimento, aproximadamente $7 \%$ das crianças apresentaram déficit de peso para idade, $8 \%$ déficit de comprimento, $6 \%$ déficit de IMC e $3 \%$ déficit de perímetro cefálico para idade.

Foram observados problemas de saúde mental aos 11 anos em 32\% (IC95\%: 30,6-33,4) dos indivíduos avaliados. Entre as meninas, a prevalência dos problemas de saúde mental foi de $29,2 \%$ e entre os meninos, de $35 \%$ ( $p=0,016)$.

A prevalência de problemas de saúde mental nas crianças aumentou linearmente com a diminuição da renda familiar, escolaridade e idade materna (Tabela 1). Observou-se uma maior proporção de problemas de saúde mental entre crianças cujas famílias tinham uma renda menor ou igual a 1 salário mínimo, filhos de mães menores de 20 anos de idade, com escolaridade entre 1-4 anos, cor da pele negra/mestiça, sem marido ou companheiro, multíparas, fumantes e consumidoras de álcool durante a gravidez (Tabela 1). Crianças com peso ao nascer $<2.500 \mathrm{~g}$ e nascidas de parto vaginal apresentaram maior frequência de problemas de saúde mental aos 11 anos (Tabela 2).

A Tabela 3 apresenta a análise bruta e ajustada das associações entre índices antropométricos ao nascer, RCIU e peso ao nascer com os problemas de saúde mental aos 11 anos. Na análise bruta, observou-se que todas as variáveis relacionadas com o tamanho ao nascimento estiveram associadas com os problemas de saúde mental aos 11 anos. Aqueles indivíduos que nasceram com déficit de peso para idade, déficit de comprimento para idade, déficit de IMC para idade e déficit de perímetro cefálico para idade, assim como aqueles que apresentaram RCIU ou peso ao nascer $<2.500 \mathrm{~g}$, tiveram mais riscos de apresentar problemas de saúde mental aos 11 anos quando comparados com aqueles indivíduos com índices antropométricos adequados ao nascimento, aqueles sem restrição ou com peso ao nascer $\geq$ $2.500 \mathrm{~g}$, respectivamente.

$\mathrm{Na}$ análise multivariável, quando ajustada para as variáveis incluídas no modelo 1 (potenciais variáveis de confusão coletadas no período perinatal), verificou-se que recém-nascidos com déficit de peso para idade e déficit de IMC para idade ao nascer apresentaram, respectivamente, $27 \%$ (razão de prevalência - RP = 1,27; IC95\%: 1,07-1,49) e 29\% (RP = 1,29; IC95\%: 1,10-1,51) mais riscos de desenvolver problemas de saúde mental aos 11 anos do que os nascidos dentro do padrão normal de tamanho ao nascer. Por outro lado, crianças que nasceram com escore- $\mathrm{Z}$ de IMC para idade e de perímetro cefálico ao nascer maior que +2 DP apresentaram, respectivamente, 34\% (RP = 1,34; IC95\%: 1,06-1,71) e $19 \%(\mathrm{RP}=1,19$; IC95\%: 1,01-1,40) mais riscos de desenvolver problemas de saúde mental que os nascidos dentro do padrão normal de tamanho ao nascer. $\mathrm{O}$ ajuste para a variável que descreve a saúde mental da mãe (modelo 2) não modificou de forma importante os achados anteriormente mencionados.

\section{Discussão}

O presente estudo sugere que crianças com déficit ou excesso do tamanho ao nascimento apresentaram um maior risco de desenvolver problemas de saúde mental aos 11 anos. Após o ajuste para variáveis maternas e perinatais, o déficit de peso para idade, o déficit e o excesso de IMC para idade, além do excesso de perímetro cefálico ao nascimento, estiveram associados 
Descrição geral dos indivíduos estudados e distribuição dos problemas de saúde mental aos 11 anos, segundo características maternas, socioeconômicas, demográficas, história reprodutiva e saúde mental. Pelotas, Rio Grande do Sul, Brasil, 1993.

\begin{tabular}{|c|c|c|c|}
\hline Variável [N] & n (\%) & $\begin{array}{c}\text { SDQ positivo aos } 11 \\
\text { anos } \\
\%(\text { IC95\%) }\end{array}$ & Valor de $p$ * \\
\hline Renda familiar (SM) [4.358] & & & $<0,001 \star \star$ \\
\hline$\leq 1,0$ & $798(18,3)$ & $42,5(39,0-45,9)$ & \\
\hline $1,1-3,0$ & $1.893(43,4)$ & $35,0(32,8-37,1)$ & \\
\hline $3,1-6,0$ & $1.034(23,7)$ & $26,8(24,1-29,5)$ & \\
\hline $6,1-10,0$ & $329(7,6)$ & $19,5(15,2-23,7)$ & \\
\hline$\geq 10,0$ & $304(7,0)$ & $17,4(13,2-21,7)$ & \\
\hline Escolaridade da mãe (anos) [4.351] & & & $<0,001 * \star$ \\
\hline 0 & $97(2,2)$ & $39,2(29,4-48,9)$ & \\
\hline $1-4$ & $1.107(25,4)$ & $40,7(37,8-43,5)$ & \\
\hline $5-8$ & $2.088(48,0)$ & $34,6(32,6-36,7)$ & \\
\hline$\geq 9$ & $1.059(24,4)$ & $17,2(14,0-19,5)$ & \\
\hline Idade da mãe (anos) [4.357] & & & $<0,001 \star \star$ \\
\hline$\leq 19$ & $767(17,6)$ & $41,6(38,1-45,1)$ & \\
\hline $20-34$ & $3.109(71,4)$ & $30,3(28,7-31,9)$ & \\
\hline$\geq 35$ & $481(11,0)$ & $27,7(23,6-31,7)$ & \\
\hline Cor da pele [4.356] & & & $<0,001$ \\
\hline Branca & $3.353(77,0)$ & $29,4(27,9-30,9)$ & \\
\hline Negra/Mestiça & $1.003(23,0)$ & $40,7(37,6-43,7)$ & \\
\hline Situação conjugal [4.358] & & & 0,001 \\
\hline Sem marido/Companheiro & $519(11,9)$ & $38,5(34,3-42,7)$ & \\
\hline Com marido/Companheiro & $3.839(88,1)$ & $31,1(29,7-32,6)$ & \\
\hline Fumo na gravidez [4.358] & & & $<0,001$ \\
\hline Não & $2.910(66,8)$ & $27,8(26,2-29,5)$ & \\
\hline Sim & $1.448(33,2)$ & $40,4(37,9-42,9)$ & \\
\hline Consumo de álcool [4.358] & & & 0,009 \\
\hline Não & $4.183(95,0)$ & $31,6(30,2-33,0)$ & \\
\hline $\operatorname{Sim}$ & $220(5,0)$ & $40,0(33,5-46,5)$ & \\
\hline Paridade [4.358] & & & $0,009 * \star$ \\
\hline 0 & $1.533(35,2)$ & $31,0(28,7-33,3)$ & \\
\hline 1 & $1.215(27,9)$ & $29,9(27,3-32,4)$ & \\
\hline$\geq 2$ & $1.610(36,9)$ & $34,6(32,3-36,9)$ & \\
\hline SRQ-20 positivo materno [4.336] & & & $<0,001$ \\
\hline Não & $2.599(59,9)$ & $20,6(18,9-22,1)$ & \\
\hline Sim & $1.737(40,1)$ & $49,0(46,6-51,3)$ & \\
\hline Total & $4.358(100,0)$ & $32,0(30,6-33,4)$ & \\
\hline
\end{tabular}

* Teste $\chi^{2}$ de Pearson;

** Teste $\chi^{2}$ tendência linear.

IC95\%: intervalo de 95\% de confiança; SDQ: Strengths and Difficulties Questionnaire; SM: salários mínimos;

SRQ-20: Self Reporting Questionnaire.

com problemas de saúde mental aos 11 anos. Esses achados estariam de acordo com a hipótese da importância dos determinantes precoces na origem do desenvolvimento da saúde e das doenças durante a vida adulta 1 .
A principal vantagem do presente trabalho é ser um estudo longitudinal de base populacional com alta taxa de seguimento e com informações coletadas de forma prospectiva, minimizando o viés de memória e permitindo identificar 
Descrição geral dos indivíduos estudados e distribuição dos problemas de saúde mental aos 11 anos, segundo características das crianças ao nascimento. Pelotas, Rio Grande do Sul, Brasil, 1993.

\begin{tabular}{|c|c|c|c|}
\hline Variável [N] & $\mathrm{n}(\%)$ & $\begin{array}{c}\text { SDO positivo } \\
\text { aos } 11 \text { anos } \\
\%(I C 95 \%)\end{array}$ & Valor de $p$ * \\
\hline Sexo da criança [4.357] & & & 0,016 \\
\hline Masculino & $2.122(48,7)$ & $35,0(32,9-37,0)$ & \\
\hline Feminino & $2.235(51,3)$ & $29,2(27,3-31,1)$ & \\
\hline Tipo de parto [4.358] & & & 0,001 \\
\hline Vaginal & $3.049(70,0)$ & $33,5(31,8-35,1)$ & \\
\hline Cesariana & $1.309(30,0)$ & $28,5(26,0-30,9)$ & \\
\hline Idade gestacional (semanas) [4.316] & & & $0,097 * \star$ \\
\hline$<34$ & $63(1,5)$ & $36,5(24,5-48,5)$ & \\
\hline $34-36$ & $382(8,8)$ & $35,3(30,5-40,1)$ & \\
\hline$\geq 37$ & $3.871(89,7)$ & $31,7(30,2-33,1)$ & \\
\hline Escore-z peso/idade ao nascer [4.351] & & & 0,001 \\
\hline$<-2 \mathrm{DP}$ & $291(6,7)$ & $42,3(36,6-48,0)$ & \\
\hline$\geq-2 \mathrm{DP}$ e $\leq+2 \mathrm{DP}$ & $4.004(92,0)$ & $31,3(29,8-32,7)$ & \\
\hline$>+2 \mathrm{DP}$ & $56(1,3)$ & $30,4(17,9-42,8)$ & \\
\hline Escore-z comprimento/idade ao nascer [4.311] & & & 0,006 \\
\hline$<-2 \mathrm{DP}$ & $362(8,4)$ & $39,5(34,4-44,6)$ & \\
\hline$\geq-2 \mathrm{DP}$ e $\leq+2 \mathrm{DP}$ & $3.874(89,9)$ & $31,3(29,8-32,7)$ & \\
\hline$>+2 \mathrm{DP}$ & $75(1,7)$ & $30,7(20,0-41,3)$ & \\
\hline Escore-z IMC/idade ao nascer [4.302] & & & $<0,001$ \\
\hline$<-2 \mathrm{DP}$ & $268(6,2)$ & $42,9(36,9-48,9)$ & \\
\hline$\geq-2 \mathrm{DP} e \leq+2 \mathrm{DP}$ & $3.932(91,4)$ & $31,2(29,7-32,6)$ & \\
\hline$>+2 \mathrm{DP}$ & $102(2,4)$ & $35,3(25,9-44,7)$ & \\
\hline Escore-z perímetro cefálico/idade ao nascer [4.328] & & & 0,029 \\
\hline$<-2 \mathrm{DP}$ & $129(3,0)$ & $42,6(34,0-51,3)$ & \\
\hline$\geq-2 \mathrm{DP} e \leq+2 \mathrm{DP}$ & $3.920(90,6)$ & $31,6(30,2-33,1)$ & \\
\hline$>+2 \mathrm{DP}$ & $279(6,4)$ & $33,0(27,4-38,5)$ & \\
\hline $\mathrm{RCIU}[3.896]$ & & & $<0,001$ \\
\hline Não & $2.992(76,8)$ & $29,8(28,2-31,5)$ & \\
\hline Sim & $904(23,2)$ & $36,1(32,9-39,2)$ & \\
\hline Peso ao nascer (g) [4.351] & & & 0,001 \\
\hline$<2.500$ & $365(8,4)$ & $37,5(32,5-42,5)$ & \\
\hline $2.500-3.499$ & $2.814(64,7)$ & $32,9(31,2-34,6)$ & \\
\hline$\geq 3.500$ & $1.172(26,9)$ & $28,1(25,5-30,6)$ & \\
\hline
\end{tabular}

* Teste $\chi^{2}$ de Pearson;

** Teste $\chi^{2}$ de tendência linear.

DP: desvio-padrão; IC95\%: intervalo de 95\% de confiança; IMC: índice de massa corporal; RCIU: restrição do crescimento intrauterino; SDQ: Strengths and Difficulties Questionnaire.

a temporalidade das associações, ainda pouco estudadas em países de renda média ou baixa. Embora a taxa de perdas fosse baixa, estas foram mais frequentes em famílias de maior escolaridade e maior renda, famílias que em todos os acompanhamentos foram as mais difíceis de convencer para participar e de ser entrevistadas.
Outra vantagem é a utilização de um questionário padronizado e validado no Brasil para a avaliação de problemas de saúde mental 24,25. Por ser o SDQ um instrumento de screening (rastreamento) com uma especificidade relativamente baixa em comparação com um teste com fins diagnósticos, a prevalência de problemas de 
Análise bruta e ajustada da associação entre índices antropométricos ao nascer, restrição do crescimento intrauterino (RCIU) e peso ao nascer com problemas de saúde mental aos 11 anos. Pelotas, Rio Grande do Sul, Brasil, 1993.

\begin{tabular}{|c|c|c|c|}
\hline \multirow[t]{2}{*}{ Variável } & \multirow[t]{2}{*}{ Análise bruta } & \multicolumn{2}{|c|}{ Análise ajustada } \\
\hline & & Modelo 1 & Modelo 2 \\
\hline Escore-z peso/idade ao nascer & $p<0,001$ & $p=0,013$ & $p<0,001$ \\
\hline$<-2 \mathrm{DP}$ & $1,35(1,17-1,56)$ & $1,27(1,07-1,49)$ & $1,32(1,13-1,55)$ \\
\hline$\geq-2 \mathrm{DP} e \leq+2 \mathrm{DP}$ & Referência $(1,00)$ & Referência $(1,00)$ & Referência $(1,00)$ \\
\hline$>+2 \mathrm{DP}$ & $0,97(0,65-1,45)$ & $1,20(0,83-1,72)$ & $1,27(0,89-1,78)$ \\
\hline Escore-z comprimento/idade ao nascer & $p=0,003$ & $p=0,278$ & $p=0,323$ \\
\hline$<-2$ DP & $1,26(1,10-1,45)$ & $1,12(0,97-1,29)$ & $1,09(0,95-1,25)$ \\
\hline$\geq-2 \mathrm{DP} e \leq+2 \mathrm{DP}$ & Referência $(1,00)$ & Referência $(1,00)$ & Referência $(1,00)$ \\
\hline$>+2 \mathrm{DP}$ & $0,98(0,69-1,38)$ & $1,11(0,80-1,52)$ & $1,13(0,84-1,53)$ \\
\hline Escore-z IMC/idade ao nascer & $p=0,004$ & $p<0,001$ & $p<0,001$ \\
\hline$<-2 \mathrm{DP}$ & $1,38(1,19-1,59)$ & $1,29(1,10-1,51)$ & $1,31(1,13-1,51)$ \\
\hline$\geq-2 \mathrm{DP}$ e $\leq+2 \mathrm{DP}$ & Referência $(1,00)$ & Referência $(1,00)$ & Referência $(1,00)$ \\
\hline$>+2 \mathrm{DP}$ & $1,13(0,87-1,48)$ & $1,34(1,06-1,71)$ & $1,30(1,02-1,64)$ \\
\hline Escore-z perímetro cefálico/idade ao nascer & $p=0,016$ & $p=0,021$ & $p=0,002$ \\
\hline$<-2 \mathrm{DP}$ & $1,35(1,10-1,66)$ & $1,25(0,99-1,57)$ & $1,34(1,09-1,65)$ \\
\hline$\geq-2 \mathrm{DP} e \leq+2 \mathrm{DP}$ & Referência $(1,00)$ & Referência $(1,00)$ & Referência $(1,00)$ \\
\hline$>+2 \mathrm{DP}$ & $1,04(0,88-1,24)$ & $1,19(1,01-1,40)$ & $1,20(1,02-1,42)$ \\
\hline $\mathrm{RCIU}$ & $p<0,001$ & $p=0,143$ & $p=0,357$ \\
\hline Não & Referência $(1,00)$ & Referência $(1,00)$ & Referência $(1,00)$ \\
\hline $\operatorname{Sim}$ & $1,21(1,09-1,34)$ & $1,08(0,97-1,19)$ & $1,05(0,95-1,15)$ \\
\hline Peso ao nascer (g) & $p=0,002$ & $p=0,106$ & $p=0,081$ \\
\hline$<2.500$ & $1,34(1,14-1,57)$ & $1,15(0,96-1,38)$ & $1,19(1,00-1,42)$ \\
\hline $2.500-3.499$ & $1,17(1,05-1,30)$ & $1,07(0.97-1,19)$ & $1,05(0,95-1,16)$ \\
\hline$\geq 3.500$ & Referência $(1,00)$ & Referência $(1,00)$ & Referência $(1,00)$ \\
\hline
\end{tabular}

DP: desvio-padrão; IC95\%: intervalo de 95\% de confiança; IMC: índice de massa corporal; modelo 1: ajustado para renda familiar, escolaridade da mãe, idade da mãe ao nascimento, cor da pele, estado civil, fumo materno e consumo de álcool durante a gravidez, paridade, sexo da criança, tipo de parto e idade gestacional; modelo 2: ajustado para modelo 1 e problemas de saúde mental da mãe avaliado pelo Self Reporting Questionnaire (SRQ-20) no acompanhamento dos 11 anos. Nota: valor de p por meio do teste de Wald para heterogeneidade.

saúde mental encontrada na população (32\%) foi possivelmente superestimada. Um estudo prévio que comparou o desempenho do SDQ frente a uma entrevista diagnóstica psiquiátrica, observou que a prevalência de transtornos mentais avaliados por esta última foi três vezes mais baixa que a prevalência encontrada pelo SDQ ${ }^{32}$. Embora o ideal fosse usar um instrumento diagnóstico que forneça uma prevalência mais real dos problemas de saúde mental na população, isto nem sempre é possível em estudos epidemiológicos. Apesar de alta, essa prevalência é similar, ou até mais baixa, do que a encontrada em outros estudos epidemiológicos com crianças e adolescentes brasileiros que utilizaram o escore total do SDQ versão para pais 33,34 .
Dentre as limitações do estudo, aponta-se a aplicação do questionário apenas à mãe, sem ter a informação dos professores ou do próprio indivíduo. Não se pode descartar também a ocorrência de confusão residual, visto que pode haver outros fatores que não foram coletados e/ou analisados no presente trabalho (outras medidas de posição socioeconômica, características do ambiente familiar e interação entre pais e filhos, assim como o IMC materno pré-gestacional) que poderiam agir como confundidores na associação entre tamanho ao nascer com os problemas de saúde mental aos 11 anos. Salienta-se que apesar das associações encontradas, algumas delas ficaram no limiar da significância estatística e algumas medidas de efeito não foram de grande magnitude (riscos relativos entre 1,19 e 
1,44). Outra limitação é o fato de nossa análise não diferenciar os problemas de saúde mental, os quais podem ter etiologias diferentes. Apesar disso, a abordagem do ciclo vital tem utilizado várias vezes o desfecho "problemas de saúde mental" em geral, maximizando nosso poder de comparabilidade dos achados com a comunidade científica.

Poucos estudos de coorte de base populacional foram realizados em países de renda média ou baixa com o objetivo de estudar as associações entre crescimento fetal e tamanho ao nascer com os problemas de saúde mental em etapas posteriores da vida. É difícil a comparação dos resultados encontrados com aqueles de estudos de coortes de países de renda alta pelas diferenças socioeconômicas e culturais e pelo impacto que elas podem ter na saúde mental da população, No entanto, mesmo com diferentes metodologias entre estudos realizados em diversos locais, foi possível comparar nossos resultados com os de estudos publicados previamente.

Pesquisas em países de renda alta têm evidenciado associação entre BPN e transtornos de comportamento e depressão em etapas mais tardias da vida. Thomas et al. 10, utilizando dados da coorte ALSPAC, evidenciaram associação entre BPN e a presença de sintomas semelhantes à psicose aos 12 anos. Gale \& Martyn 35 evidenciaram que mulheres com peso inferior a $3 \mathrm{~kg}$ no nascimento tiveram um risco aumentado de depressão aos 26 anos de idade. Outros autores encontraram associação entre BPN e estresse psicológico na idade adulta para ambos os sexos 16,36. O BPN também foi associado a um maior risco de esquizofrenia 37,38 , transtorno depressivo em homens 17 e maior risco de suicídio na vida adulta 15. Em um estudo realizado na coorte de Pelotas do ano de 1982, não se encontrou associação entre o BPN e transtornos mentais comuns em indivíduos aos 22 anos de vida 39 .

Consistente com o estudo de Alati et al. 40, o presente trabalho encontrou uma associação significativa entre déficit de peso para idade ao nascer e problemas de saúde mental aos 11 anos. Esses investigadores, utilizando dados de 4.971 participantes do estudo de coorte de gestantes Mater University Study of Pregnancy (MUSP) de 1981, de Brisbane (Austrália), observaram que crianças no quintil mais baixo e no quintil mais alto de escore-z de peso para idade, apresentaram maior risco de ansiedade e/ou depressão e sintomas de problemas sociais aos 14 anos.

O presente estudo verificou que o déficit de IMC para idade ao nascer esteve associado com os problemas de saúde mental aos 11 anos. Embora não tenha sido encontrado nenhum estudo que avaliasse o IMC ao nascimento e problemas de saúde mental em crianças ou adolescentes, nossos achados foram consistentes com os apresentados em pesquisas com adultos. Lahti et al. ${ }^{19}$ e Räikkönen et al. ${ }^{20}$, no estudo de coorte de nascimentos de Helsinki de 1934-1944, reportaram uma associação inversa entre IMC ao nascer com transtornos de ansiedade e com atitude de hostilidade na vida adulta. Na mesma linha, Nilsson et al. 41, na coorte sueca de homens nascidos entre 1973-1975, encontraram que quanto maior o peso e IMC ao nascer, melhor foi o escore de funcionamento psicológico geral.

O estudo realizado por Wiles et al. 9, utilizando dados de 4.813 crianças nascidas entre 19981999 pertencentes à coorte de ALSPAC em Bristol (Reino Unido), avaliou a associação entre o comprimento ao nascer com os problemas de saúde mental aos 7 anos, encontrando que o aumento em um DP no comprimento ao nascer esteve associado com uma redução de $14 \%$ na probabilidade de estar no tercil superior de dificuldades comportamentais aos 7 anos, embora com uma associação limítrofe. Nosso estudo, contudo, não mostrou associação entre escore-z de comprimento para idade ao nascimento com problemas de saúde mental aos 11 anos.

Observou-se que recém-nascidos com escores-z de IMC ao nascer maior que +2 DP tiveram $34 \%$ mais riscos de apresentar problemas de saúde mental aos 11 anos. Esses resultados sugerem que doenças que levam à macrosomia fetal, como a diabetes gestacional 42,43 , poderiam influenciar o desenvolvimento de problemas de saúde mental em etapas posteriores. Alguns estudos têm evidenciado associação entre diabetes gestacional e transtornos de comportamento, menor capacidade cognitiva e maior hospitalização por transtornos no neuro-desenvolvimento em crianças de mulheres com esta doença 44,45 .

Encontramos também um maior risco de problemas de saúde mental aos 11 anos em recém-nascidos com escores-z de perímetro cefálico maior que +2 DP. Não entanto, essa associação foi limítrofe (IC95\%: 1,02-1,42), o que sugere a presença de confusão residual. O perímetro cefálico no extremo superior poderia ser um indicador de distúrbios genéticos ou malformações que per se fossem responsáveis por problemas de comportamento em etapas mais tardias, ou também poderia estar correlacionado ao maior tamanho corporal que foi associado significativamente ao desenvolvimento de problemas de saúde mental neste estudo.

Os achados deste estudo sugerem que fatores que afetam o tamanho ao nascer, tanto produzindo déficit como excesso, poderiam estar envolvidos na aparição dos problemas de saúde mental 
nas etapas posteriores da vida. Pesquisas prévias demonstraram a associação entre fumo materno ou alcoolismo durante a gravidez e problemas do comportamento na infância e adolescência $46,47,48$. No nosso trabalho, após ajuste para fumo materno e consumo de álcool na gravidez, as associações entre déficit de peso e IMC para idade ao nascimento se mantiveram significativas.

Estudos prospectivos têm evidenciado que problemas de saúde mental durante as primeiras etapas da vida aumentam a vulnerabilidade dos indivíduos para o desenvolvimento destes transtornos na vida futura 49,50,51. A prevenção de problemas mentais durante as diferentes fases da vida é um desafio importante para a saú- de pública, porém, o pouco conhecimento sobre suas causas dificulta a prevenção primária. No entanto, a identificação de vulnerabilidades precoces, como as descritas no presente estudo, pode colaborar para que este grupo de crianças receba atenção especial dos serviços de saúde, o que possivelmente irá facilitar a detecção e tratamento precoce dos problemas mentais nas crianças e diminuir suas consequências nas fases posteriores da vida. Futuras investigações epidemiológicas com delineamento longitudinal e com informação detalhada sobre acontecimentos ocorridos no período perinatal serão necessárias para continuar a elucidar as origens dos problemas de saúde mental dos indivíduos.

\section{Resumo}

O objetivo foi avaliar a associação entre tamanho ao nascer e problemas de saúde mental aos 11 anos na Coorte de Nascimentos de Pelotas, Rio Grande do Sul, Brasil, de 1993. Foram pesados e medidos ao nascer 4.358 recém-nascidos. Avaliou-se problemas de saúde mental com o questionário de capacidades e dificuldades (Strengths and Difficulties Questionnaire - SDQ). A prevalência de problemas de saúde mental foi de 32\% (IC95\%: 31-33). Na análise ajustada, os 291 (6,7\%) recém-nascidos com escore- $z$ de peso/idade e os 268 (6,2\%) com índice de massa corporal (IMC)/idade $<-2$ DP tiveram, respectivamente, 27\% (IC95\%: 7-49) e 29\% (IC95\%: 10-51) maior risco de apresentar problemas de saúde mental aos 11 anos quando comparados com aqueles com escore normal. Os 102 (2,43\%) recém-nascidos com escore-z de IMC e os 279 (6,4\%) com perímetro cefálico/idade $>+2$ DP tiveram, respectivamente, 34\% (IC95\%: 6-71) e 19\% (IC95\%: 1-40) maior risco de apresentar esses problemas se comparados com aqueles com escore normal. Os resultados sugerem que fatores ocorridos na gestação e refletidos nas medidas de tamanho ao nascer podem ocasionar problemas de saúde mental em etapas tardias.

Desenvolvimento Fetal; Saúde Mental; Antropometria

\section{Colaboradores}

A. Matijasevich, E. A. G. Gallo e S. C. Dumith participaram da análise dos dados, redação do manuscrito e aprovação da versão final. L. Anselmi, M. Scazufca, A. M. B. Menezes e P. C. Hallal colaboraram na interpretação dos achados, redação do artigo e aprovação da versão final.

\section{Agradecimentos}

O estudo de coorte de 1993 é apoiado pela fundação Wellcome Trust. As fases iniciais do estudo de coorte foram financiadas pela União Europeia, pelo Programa Nacional para Centros de Excelência (PRONEX), pelo Conselho Nacional de Desenvolvimento Científico e Tecnológico (CNPq) e pelo Ministério da Saúde do Brasil. 


\section{Referências}

1. Barker DJP. The developmental origins of adult disease. J Am Coll Nutr 2004; 23(6 Suppl):588S-95S.

2. De Boo HA, Harding JE. The developmental origins of adult disease (Barker) hypothesis. Aust N Z J Obstet Gynaecol 2006; 46:4-14.

3. Schlotz W, Phillips DI. Fetal origins of mental health: evidence and mechanisms. Brain Behav Immun 2009; 23:905-16.

4. Eriksson JG, Forsen TJ, Osmond C, Barker DJP. Pathways of infant and childhood growth that lead to type 2 diabetes. Diabetes Care 2003; 26:3006-10.

5. Strupp BJ, Levitsky DA. Enduring cognitive effects of early malnutrition: a theoretical reappraisal. J Nutr 1995; 125(8 Suppl):2221S-32S.

6. Levitsky DA, Strupp BJ. Malnutrition and the brain: changing concepts, changing concerns. J Nutr 1995; 125(8 Suppl):2212S-20S.

7. Walker SP, Grantham-Mcgregor SM, Powell CA, Chang SM. Effects of growth restriction in early childhood on growth, IQ, and cognition at age 11 to 12 years and the benefits of nutritional supplementation and psychosocial stimulation. J Pediatr 2000; 137:36-41.

8. Kajantie E. Fetal origins of stress-related adult disease. Ann N Y Acad Sci 2006; 1083:11-27.

9. Wiles NJ, Peters TJ, Heron J, Gunnell D, Emond A, Lewis G, et al. Fetal growth and childhood behavioral problems: results from the ALSPAC cohort. Am J Epidemiol 2006; 163:829-37.

10. Thomas K, Harrison G, Zammit S, Lewis G, Horwood J, Heron J, et al. Association of measures of fetal and childhood growth with non-clinical psychotic symptoms in 12-year-olds: the ALSPAC cohort. Br J Psychiatry 2009; 194:521-6.

11. Wahlbeck K, Forsen T, Osmond C, Barker DJP, Eriksson JG. Association of schizophrenia with low maternal body mass index, small size at birth, and thinness during childhood. Arch Gen Psychiatry 2001; 58:48-52.

12. Gunnell D, Harrison G, Whitley E, Lewis G, Tynelius P, Rasmussen F. The association of fetal and childhood growth with risk of schizophrenia. Cohort study of 720,000 Swedish men and women. Schizophr Res 2005; 79:315-22.

13. Saigal S. Follow-up of very low birthweight babies to adolescence. Semin Neonatol 2000; 5:107-18.

14. Brown AS, Susser ES, Lin SP, Neugebauer R, Gorman JM. Increased risk of affective disorders in males after second trimester prenatal exposure to the Dutch hunger winter of 1944-45. Br J Psychiatry 1995; 166:601-6.

15. Barker DJP, Osmond C, Rodin I, Fall CHD, Winter PD. Low weight gain in infancy and suicide in adult life. BMJ 1995; 311:1203-08.

16. Cheung YB, Khoo KS, Karlberg J, Machin D. Association between psychological symptoms in adults and growth in early life: longitudinal follow up study. BMJ 2002; 325:749.

17. Thompson C, Syddall H, Rodin IAN, Osmond C, Barker DJP. Birth weight and the risk of depressive disorder in late life. Br J Psychiatry 2001; 179:450-5.
18. Hack M, Schluchter M, Cartar L, Rahman M, Cuttler L, Borawski E. Growth of very low birth weight infants to age 20 years. Pediatrics 2003; 112(1 Pt 1): 30-8.

19. Lahti J, Räikkönen K, Kajantie E, Heinonen K, Pesonen AK, Järvenpää AL, et al. Small body size at birth and behavioural symptoms of ADHD in children aged five to six years. J Child Psychol Psychiatry 2006; 47:1167-74.

20. Räikkönen K, Pesonen A-K, Heinonen K, Lahti J, Kajantie E, Forsen T, et al. Infant growth and hostility in adult life. Br J Psychiatry 2008; 70:306-13.

21. Ministério da Saúde. Cadernos de informações de saúde dos municípios do Rio Grande do Sul. Brasília: Ministério da Saúde; 2009.

22. Victora CG, Hallal PC, Araújo CL, Menezes AM, Wells JC, Barros FC. Cohort profile: the 1993 Pelotas (Brazil) birth cohort study. Int J Epidemiol 2008; 37:704-9.

23. Victora CG, Araújo CLP, Menezes AMB, Hallal PC, Vieira MF, Neutzling MB, et al. Methodological aspects of the 1993 Pelotas (Brazil) birth cohort study. Rev Saúde Pública 2006; 40:39-46.

24. Fleitlich-Bilyk B, Goodman R. Prevalence of child and adolescent psychiatric disorders in southeast Brazil. J Am Acad Child Adolesc Psychiatry 2004; 43:727-34.

25. Stivanin L, Scheuer CI, Assumpção Jr. FB. SDQ (Strengths and Difficulties Questionnaire): identificação de características comportamentais de crianças leitoras. Psicol Teor Pesqui 2008; 24:407-13.

26. Goodman R. Strengths and Difficulties Questionnaire. http://www.sdqinfo.org/ (acessado em 13/ Dez/2010).

27. World Health Organization. Child growth standards based on length/height, weight and age. Acta Paediatr 2006; 450:76-85.

28. Kramer MS, Platt RW, Wen SW, Joseph KS, Allen A, Abrahamowicz M, et al. A new and improved population-based Canadian reference for birth weight for gestational age. Pediatrics 2001; 108:E35.

29. Dubowitz LM, Dubowitz V, Goldberg C. Clinical assessment of gestational age in the newborn infant. J Pediatr 1970; 77:1-10.

30. Mari JJ, Williams P. A validity study of a psychiatric screening questionnaire (SRQ-20) in primary care in the city of Sao Paulo. Br J Psychiatry 1986; 148:23-6.

31. World Health Organization. A user's guide to the Self Reporting Questionnaire (SRQ). Geneva: World Health Organization; 1994.

32. Anselmi L, Fleitlich-Bilyk B, Menezes AM, Araújo CL, Rohde LA. Prevalence of psychiatric disorders in a Brazilian birth cohort of 11-year-olds. Soc Psychiatry Psychiatr Epidemiol 2010; 45:135-42.

33. Goodman R, Neves dos Santos D, Robatto Nunes AP, Pereira de Miranda D, Fleitlich-Bilyk B, Almeida Filho N. The Ilha de Maré study: a survey of child mental health problems in a predominantly African-Brazilian rural community. Soc Psychiatry Psychiatr Epidemiol 2005; 40:11-7. 
34. Rodriguez JD, Silva AA, Bettiol H, Barbieri MA, Rona RJ. The impact of perinatal and socioeconomic factors on mental health problems of children from a poor Brazilian city: a longitudinal study. Soc Psychiatry Psychiatr Epidemiol 2011; 46:381-91.

35. Gale CR, Martyn CN. Birth weight and later risk of depression in a national birth cohort. Br J Psychiatry 2004; 184:28-33.

36. Wiles NJ, Peters TJ, Leon DA, Lewis G. Birth weight and psychological distress at age $45-51$ years: results from the Aberdeen Children of the 1950s cohort study. Br J Psychiatry 2005; 187:21-8.

37. Niemi LT, Suvisaari JM, Haukka JK, Lonnqvist JK. Childhood growth and future development of psychotic disorder among Helsinki high-risk children. Schizophr Res 2005; 76:105-12.

38. Rifkin L, Lewis S, Jones P, Toone B, Murray R. Low birth weight and schizophrenia. Br J Psychiatry 1994; 165:357-62.

39. Anselmi L, Barros FC, Minten GC, Gigante DP, Horta BL, Victora CG. Prevalência e determinantes precoces dos transtornos mentais comuns na coorte de nascimentos de 1982, Pelotas, RS. Rev Saúde Pública 2008; 42 Suppl 2:26-33.

40. Alati R, Najman JM, O'Callaghan M, Bor W, Williams GM, Clavarino A. Fetal growth and behaviour problems in early adolescence: findings from the Mater University Study of Pregnancy. Int J Epidemiol 2009; 38:1390-400.

41. Nilsson PM, Nyberg P, Ostergren PO. Increased susceptibility to stress at a psychological assessment of stress tolerance is associated with impaired fetal growth. Int J Epidemiol 2001; 30:75-80.

42. Amorim MM, Leite DF, Gadelha T, Muniz AG, Melo AS, Rocha A. Fatores de risco para macrossomia em recém-nascidos de uma maternidade-escola no nordeste do Brasil. Rev Bras Ginecol Obstet 2009; 31:241-8.

43. Sheree LB, Greg RA, Hamisu MS, MaryAnn P. Macrosomic births in the United States: determinants, outcomes, and proposed grades of risk. Am J Obstet Gynecol 2003; 188:1372-8.
44. Aberg A, Westbom L. Association between maternal pre-existing or gestational diabetes and health problems in children. Acta Pædiatr 2001; 90:746-50.

45. Rizzo T, Metzger BE, Burns WJ, Burns K. Correlations between antepartum maternal metabolism and child intelligence. N Engl J Med 1991; 325:911-6.

46. Wakschlag LS, Hans SL. Maternal smoking during pregnancy and conduct problems in high-risk youth: a developmental framework. Dev Psychopathol 2002; 14:351-69.

47. Barr HM, Bookstein FL, O'Malley KD, Connor PD, Huggins JE, Streissguth AP. Binge drinking during pregnancy as a predictor of psychiatric disorders on the Structured Clinical Interview for DSM-IV in young adult offspring. Am J Psychiatry 2006; 163:1061-5.

48. Sayal K, Heron J, Golding J, Emond A. Prenatal alcohol exposure and gender differences in childhood mental health problems: a longitudinal population-based study. Pediatrics 2007; 119:426-34.

49. Anselmi L, Barros FC, Teodoro ML, Piccinini CA, Menezes AM, Araújo CL, et al. Continuity of behavioral and emotional problems from pre-school years to pre-adolescence in a developing country. J Child Psychol Psychiatry 2008; 49:499-507.

50. Costello EJ, Mustillo S, Erkanli A, Keeler G, Angold A. Prevalence and development of psychiatric disorders in childhood and adolescence. Arch Gen Psychiatry 2003; 60:837-44.

51. Caspi A, Moffitt TE, Newman DL, Silva PA. Behavioral observations at age 3 years predict adult psychiatric disorders. Longitudinal evidence from a birth cohort. Arch Gen Psychiatry 1996; 53:1033-9.

Recebido em 25/Jan/2011

Versão final reapresentada em 06/Mai/2011

Aprovado em 01/Jun/2011 